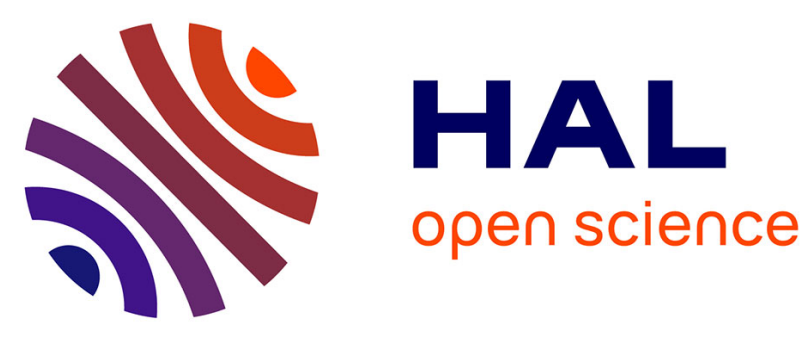

\title{
IQ Imbalance Compensation Based on Maximum SNR Estimation in Coherent QPSK Systems
}

\author{
Trung Hien Nguyen, Fausto Gomez Agis, Mathilde Gay, Luiz Anet Neto, \\ Pascal Scalart, Christophe Peucheret, Michel Joindot, Olivier Sentieys, \\ Jean-Claude Simon, Laurent Bramerie
}

\section{To cite this version:}

Trung Hien Nguyen, Fausto Gomez Agis, Mathilde Gay, Luiz Anet Neto, Pascal Scalart, et al.. IQ Imbalance Compensation Based on Maximum SNR Estimation in Coherent QPSK Systems. 16th International Conference on Transparent Optical Networks (ICTON 2014), Jul 2014, Graz, Austria. paper Tu.C1.3, 10.1109/ICTON.2014.6876406 . hal-01062978

\section{HAL Id: hal-01062978 https://hal.science/hal-01062978}

Submitted on 12 Sep 2014

HAL is a multi-disciplinary open access archive for the deposit and dissemination of scientific research documents, whether they are published or not. The documents may come from teaching and research institutions in France or abroad, or from public or private research centers.
L'archive ouverte pluridisciplinaire HAL, est destinée au dépôt et à la diffusion de documents scientifiques de niveau recherche, publiés ou non, émanant des établissements d'enseignement et de recherche français ou étrangers, des laboratoires publics ou privés. 


\title{
IQ Imbalance Compensation Based on Maximum SNR Estimation in Coherent QPSK Systems
}

\author{
Trung-Hien Nguyen ${ }^{1,2}$, Fausto Gomez-Agis ${ }^{1,2}$, Mathilde Gay ${ }^{1,2}$, Luiz Anet-Neto ${ }^{1,2}$, Pascal Scalart ${ }^{1,3}$, \\ Christophe Peucheret ${ }^{1,2}$, Michel Joindot ${ }^{1,2}$, Olivier Sentieys ${ }^{1,3}$, Jean-Claude Simon ${ }^{1,2}$, \\ and Laurent Bramerie ${ }^{1,2}$ \\ ${ }^{1}$ Université Européenne de Bretagne (UEB), 5 Boulevard Laënnec, 35000 Rennes, France \\ ${ }^{2}$ CNRS-Foton Laboratory (UMR 6082), Enssat, CS 80518, 22305 Lannion, France \\ ${ }^{3}$ INRIA/IRISA, 35000 Rennes, France \\ Tel: (0033) 29646 9118,Fax: (0033) 29646 9076, e-mail: tnguyen@enssat.fr
}

\begin{abstract}
We present a simple alternative method for the compensation of quadrature imbalance in optical quadrature phase-shift-keying (QPSK) coherent systems. The method is based on the determination and the compensation of the phase mismatch by the introduction of a relevant signal-to-noise ratio metric. The principle is validated numerically and the algorithm is validated experimentally through bit-error-rate (BER) and error vector magnitude (EVM) measurements. A $20 \mathrm{~Gb} / \mathrm{s}$ optical QPSK experiment reveals a good agreement of the proposed method with the Gram-Schmidt orthogonalization procedure (GSOP). Moreover, the robustness of both methods was verified with up to $30^{\circ}$ phase misalignment by comparing the signal after phase imbalance compensation to that without compensation. A $10 \%$ reduction of EVM is achieved with our method for a high phase misalignment of $30^{\circ}$.
\end{abstract}

Keywords: in-phase/quadrature imbalance, digital signal processing, fiber optic communications, coherent communications.

\section{INTRODUCTION}

Over the last decade, there has been a renewed interest in coherent optical communication systems because of their improved receiver sensitivity and spectral efficiency, and ability to mitigate transmission impairments in the digital domain [1]. In particular, the quadrature phase-shift-keying (QPSK) format has been the object of intensive investigations and $100 \mathrm{~Gb} / \mathrm{s}$ transmission systems using this format are now commercially available. Ideally, in a QPSK system, the in-phase and quadrature components of the optical field should be orthogonal to each other. However, hardware implementation imperfections such as incorrect bias-points settings in the modulator, imperfect splitting ratio of couplers, photodiodes responsivity mismatch and misadjustment of polarization splitters in the optical coherent receiver can create amplitude and phase imbalance, known as quadrature imbalance (or IQ imbalance), which destroys the orthogonality of the received signal [2]. Since digital signal processing (DSP) circuits are becoming increasingly faster, providing simple and efficient compensation of linear and, possibly, non-linear impairments, it is important to assess their potential for the compensation of this detrimental loss of orthogonality in the receiver. It has already been demonstrated that IQ imbalance in coherent QPSK systems can be corrected by applying different methods such as the Gram-Schmidt orthogonalization procedure (GSOP) [3], the ellipse correction method (EC) [4] or an IQ compensation based on the constant modulus algorithm [5].

In this paper, we propose an alternative method for IQ imbalance compensation based on the definition and estimation of a suitable signal-to-noise ratio (SNR) metric for the detected signal. This approach, called maximum SNR estimation method (MSEM), provides an attractive alternative to existing algorithms thanks to its reduced complexity. Indeed, the proposed method requires simple mathematical functions such as exponentiation or division, whereas the GSOP method requires other more complex mathematical functions such as square root operation, which make its implementation in field-programmable gate array (FPGA) circuits more complex. Therefore the proposed algorithm is suitable for real-time signal processing. Along with the EC method, our method can predict the phase mismatch between in-phase and quadrature components, which is not possible with other published approaches $[3,5]$. We also experimentally compare the accuracy of our method to the GSOP approach by evaluating the bit-error-rate (BER) and the error vector magnitude (EVM) on the compensated constellations. The performance of the proposed MSEM method matches that of the GSOP, while its implementation complexity is reduced.

\section{PRINCIPLE OF THE METHOD}

In our study, we focus only on phase misalignment since amplitude imbalance can be corrected at the hardware level [6]. At the output of a phase-diversity coherent receiver, we can define the in-phase (I) and quadrature (Q) terms by the following equations: 


$$
\begin{aligned}
& \mathbf{I} \propto \operatorname{Re}\left\{E_{S} E_{L O}^{*}\right\} \\
& \mathbf{Q} \propto \operatorname{Im}\left\{E_{S} E_{L O}^{*} \cdot \mathrm{e}^{j \phi_{m i s}}\right\}
\end{aligned}
$$

where $E_{S}$ and $E_{L O}$ represent the fields of the signal (S) and local oscillator (LO) at the inputs to the $90^{\circ}$ hybrid in the receiver, $\mathrm{Re}$ and $\mathrm{Im}$ denote the real and imaginary parts of the beating products between $\mathrm{S}$ and LO, respectively. $\phi_{\text {mis }}$ represents the phase imbalance between the complex quadratures. In the ideal case $\phi_{\text {mis }}=0$, whereas imperfections in the implementation of the QPSK transmission system can lead to $\phi_{\text {mis }} \neq 0$ and consequently to a loss of orthogonality. It should be noted that $\phi_{\text {mis }}$ is in the range $[-\pi / 2, \pi / 2]$ since the maximum phase difference between $\mathbf{I}$ and $\mathbf{Q}$ components is $\pi$ radians. The MSEM method consists in considering an additional phase parameter in the quadrature term, $\phi_{v a r}$, so that $\mathbf{Q}_{\mathrm{n}}=\operatorname{Im}\left\{r_{x} \cdot \exp \left(j \cdot \phi_{v a r}\right)\right\}$, where $r_{x}=\mathbf{I}+j \cdot \mathbf{Q}$ is the received signal. The relevant estimated SNR metric is defined by:

$$
S N R_{e s t}=\frac{E^{2}\left(|r|^{2}\right)}{\sigma_{|r|^{2}}^{2}}
$$

where $\mathrm{E}(\cdot)$ and $|\cdot|$ are the mean and modulus operators, respectively. $\sigma_{|r|^{2}}^{2}$ denotes the variance of the square modulus of the modified signal, $r=\mathbf{I}+j \cdot \mathbf{Q}_{\mathrm{n}}$. In practical implementations, the IQ imbalance due to transmitter or receiver imperfections should be sufficiently well controlled so that the value of the IQ imbalance remains in the range $[-\pi / 4, \pi / 4]$. In the presence of IQ imbalance, the square modulus of $r$ will decrease, inducing a decrease in the proposed SNR metric. Consequently, by evaluating the SNR for $\phi_{v a r}$ varying between $-\pi / 4$ to $\pi / 4$, the IQ imbalance term will correspond to $\phi_{m i s}=-\phi_{v a r}$, where $\phi_{v a r}$ is the value that maximizes the SNR.

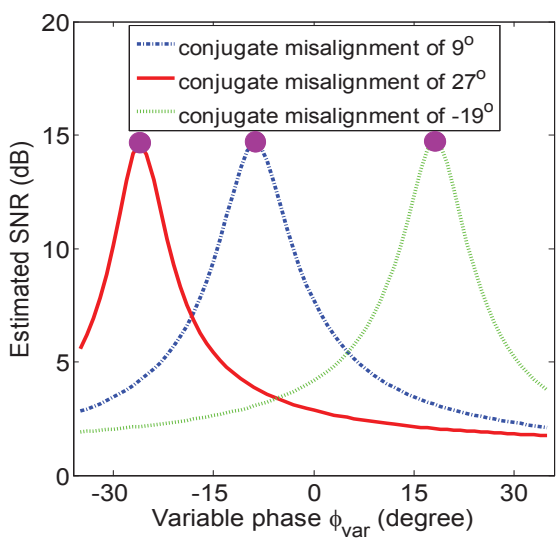

Figure 1. Numerical SNR estimation for different variable phase values using the proposed MSEM method.

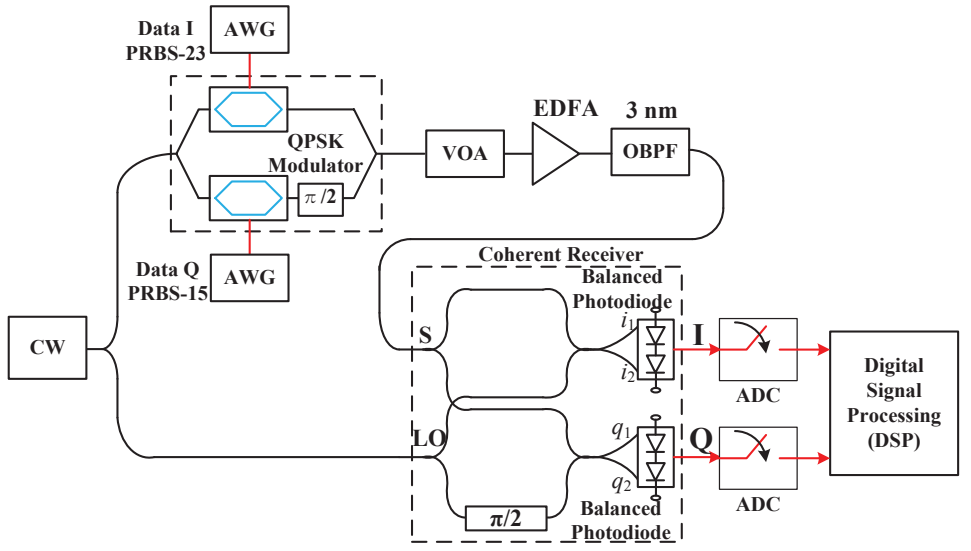

Figure 2. Experimental setup for characterizing the quadrature imbalance compensation in a QPSK coherent system.

\section{NUMERICAL VALIDATION}

The principle of the proposed method is first validated using numerical simulations. The optical field at the output of the QPSK transmitter is represented according to:

$$
E_{\text {out }}=\frac{E_{\text {in }}}{2}\left[\cos \left(\frac{\pi V_{I}}{2 V_{\pi}}\right)+j \cos \left(\frac{\pi V_{Q}}{2 V_{\pi}}\right)\right]
$$

where $V_{I}$ and $V_{Q}$ are the driving voltages applied to the I and Q ports of the quadrature modulator, respectively. Both Mach-Zehnder modulators (MZMs) are biased at null transmission points and $V_{\pi}$ is their half-wave voltage. The laser source linewidth, $\Delta v$ is assumed to be equal to $200 \mathrm{kHz}$. The effect of phase noise, $\varphi_{n}$, is modeled as a discrete time random walk $\varphi_{n}=\varphi_{n}+\Delta_{n}$, in which $\Delta_{n}$ is a Gaussian random variable with zero mean and variance $2 \pi \cdot \Delta v \cdot T_{B}$, where $T_{B}$ is the QPSK symbol duration. The phase mismatch is emulated at the receiver side by varying the phase difference between the $\mathbf{I}$ and $\mathbf{Q}$ components, which causes the loss of orthogonality. The noise generated during transmission is modelled as additive white Gaussian noise (AWGN). Considering a 20 $\mathrm{Gb} / \mathrm{s}$ QPSK signal, Fig. 1 represents numerical estimations of the SNR metric previously defined as a function of $\phi_{v a r}$ for different predefined phase mismatches of $-19^{\circ}, 9^{\circ}$ and $27^{\circ}$ at a degraded electrical SNR of $12 \mathrm{~dB}$ obtained from addition of Gaussian noise. It is confirmed that the maximum estimated SNRs occur at $\phi_{v a r}=-\phi_{\text {mis }}$ for all values of phase misalignment, which validates the method. 


\section{EXPERIMENTAL SETUP AND PERFORMANCE OF THE MSEM METHOD}

The impact of non-orthogonality between I and Q quadratures is then experimentally investigated using the optical QPSK coherent system shown in Fig. 2. To simplify the setup and avoid any impact from other functionalities implemented in the digital signal processing (DSP), the same laser is used as transmitter and LO at the receiver in order to ease the carrier frequency offset compensation. The estimated linewidth of this optical source tuned to a wavelength of $1540 \mathrm{~nm}$ is about $100 \mathrm{kHz}$. To facilitate BER measurements, two pseudorandom binary sequences (PRBSs) with lengths of $2^{15}-1$ and $2^{23}-1$ are used as inputs to an IQ modulator, resulting in a 10 Gbaud non return-to-zero (NRZ)-QPSK signal at the modulator output. The bit sequences are synthesized using arbitrary waveform generators (AWG). An adjustable phase imbalance can be easily introduced by tuning the voltage applied to the phase-shifter controlling the relative phase between the two arms of the IQ modulator. The use of a variable optical attenuator (VOA) followed by an erbium-doped fiber amplifier (EDFA) and a $3 \mathrm{~nm}$ optical bandpass filter (OBPF) allows the adjustment of the optical signal-to-noise ratio (OSNR). At the receiver side, an optical $90^{\circ}$ hybrid (100 Gb/s DP-QPSK integrated receiver) mixes the QPSK signal and the LO. The in-phase and quadrature components are then detected by balanced photodiodes with a bandwidth of $32 \mathrm{GHz}$. Finally, the electrical output signals are acquired by a real time oscilloscope with electrical bandwidth of $16 \mathrm{GHz}$. After data acquisition at $20 \mathrm{GS} / \mathrm{s}$, postprocessing is performed offline using the Matlab environment.
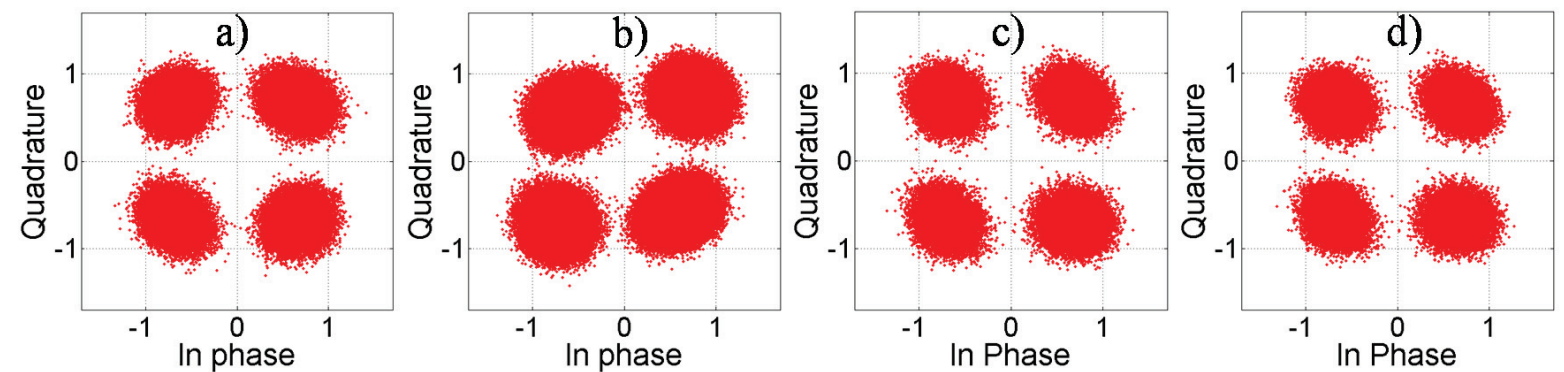

Figure 3. Experimental QPSK constellations at $12 \mathrm{~dB}$ OSNR: a) without IQ imbalance, b) with IQ imbalance of $15^{\circ}$, c) recovered using the GSOP method, d) recovered using the proposed MSEM method.

In the first step of digital processing, we use a well-known adaptive finite impulse response (FIR) filter [7] as an equalizer. The equalizer operates at twice the symbol rate to be able to adaptively compensate environmental changes such as temperature. After stabilization of the feedback loop of the equalizer, the following process is performed with 1 sample per symbol by decimating the symbol rate to $10 \mathrm{GS} / \mathrm{s}$. The algorithms for IQ imbalance compensation are applied on blocks of 1000 samples. The widely-used Viterbi-Viterbi algorithm based phase estimation is performed on blocks of 10 samples, enabling to reconstruct the constellations. Moreover, the phase ambiguity is removed by processing the first 5000 samples to minimize the BER. Finally, the decoded bit sequence is compared to the transmitted one to determine the BER over 4 million samples.

Figure 3(a) shows the experimental constellation without IQ imbalance when the signal OSNR is $12 \mathrm{~dB}$ (over $0.1 \mathrm{~nm})$. Figure 3(b) represents the constellation of the degraded signal with $17^{\circ}$ phase mismatch between I and Q. This signal degradation leads to detection errors, as it will be shown later. This degraded signal is processed with the same DSP procedure that deploys either the GSOP or the proposed MSEM method, resulting in the constellations in Fig. 3(c) and Fig. 3(d), respectively. The retrieved constellations are similar to the one without IQ imbalance thanks to the quadrature imbalance corrections. In the next step, our method is experimentally validated by comparing its performance to that of the GSOP method in terms of BER and EVM.

Figure 4 presents the results of BER measurement as a function of received OSNR in different cases. The theoretical curve of a QPSK signal (circles) without IQ imbalance is plotted based on the analysis in [7]. The experimental measurement without IQ imbalance (stars) matches the theoretical trace within experimental errors. Next, the case of a phase mismatch of $17^{\circ}$ without compensation (diamonds) is plotted in the same figure. As expected, the OSNR is degraded by about $1.7 \mathrm{~dB}$ at a BER of $10^{-3}$. The BER after phase mismatch compensation using the GSOP (empty triangles) and the MSEM methods (filled triangles) are plotted. In both cases, the IQ imbalance is well compensated, reducing the power penalty close to the experimental case without IQ imbalance. The superposition of results using both compensation methods reveals their good agreement. To further investigate the accuracy of the proposed algorithm, the EVM is calculated as a function of $\phi_{\text {mis }}$ at different OSNR values (Fig. 5). It can be observed that, as the IQ imbalance increases, and when no compensation is applied (circles), the EVM increases, representing an important deviation from the optimal constellation (up to $40 \%$ variation). With both correction methods - MSEM (stars) and GSOP (diamonds) - and with an OSNR higher than $10 \mathrm{~dB}$, the EVM remains almost constant for a phase misalignment lower than $17^{\circ}$. Both methods can partially compensate higher misalignments with a reduction of the EVM variation of about $10 \%$. Finally, for an OSNR of $8 \mathrm{~dB}$, the EVM starts to increase above a misalignment of $9^{\circ}$. 


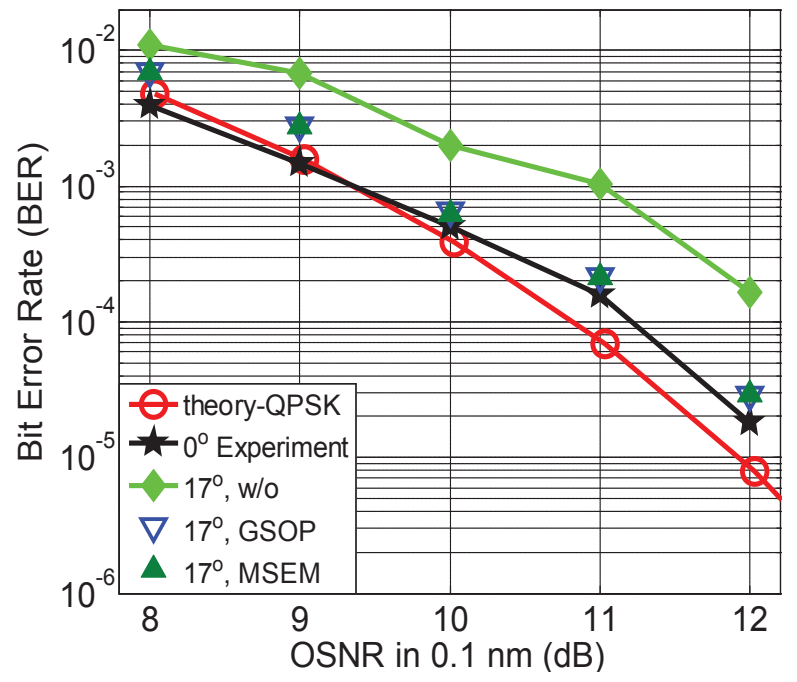

Figure 4. BER measurement as a function of OSNR (in $0.1 \mathrm{~nm}$ ) for phase misalignments of $0^{\circ}$ and $17^{\circ}$ without (w/o) any correction and with compensation using the GSOP and MSEM methods.

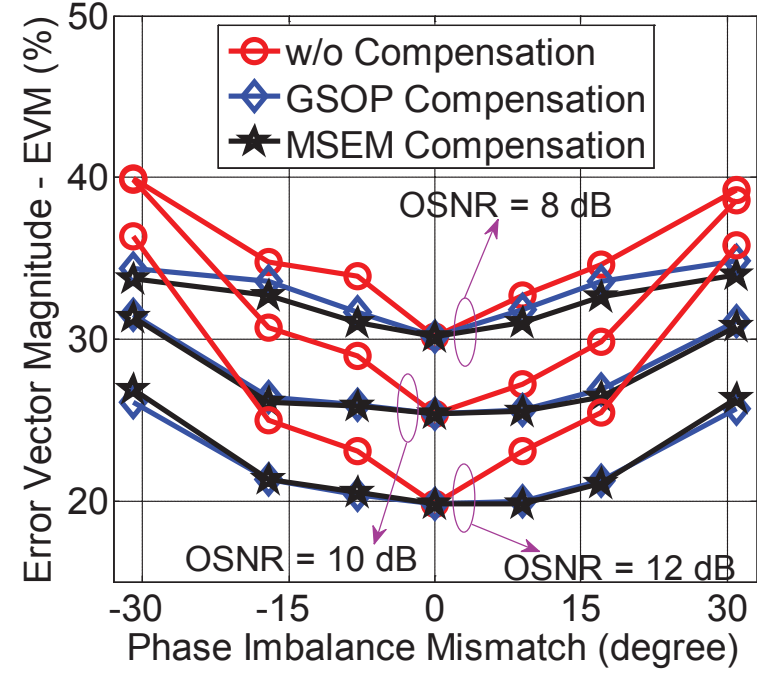

Figure 5. EVM calculation under the impact of IQ imbalance without and with compensation by the GSOP and MSEM methods.

\section{CONCLUSIONS}

We have proposed and demonstrated the MSEM method as an alternative solution for orthogonal quadrature mismatch in QPSK systems. The accuracy of this method is experimentally validated by comparing it with the GSOP method by means of BER and EVM measurements. The new compensation method provides a good correction with lower implementation complexity, even if the conjugate misalignment reaches $30^{\circ}$.

\section{ACKNOWLEDGEMENTS}

The authors thank the national projects OCELOT and PONANT, as well as the French Ministry of Research for funding this work.

\section{REFERENCES}

[1] E. Ip, A.P.T. Lau, D.J.F. Barros, and J.M. Kahn: Coherent detection in optical fiber systems, Opt. Express, vol. 16, pp. 753-791, 2008.

[2] X. Liu: Receiver sensitivity improvement in optical DQPSK and DQPSK/ASK through data-aided multisymbol phase estimation, in Proc. ECOC, Th.4.4.5, 2006.

[3] I. Fatadin et al::Compensation of quadrature imbalance in an optical QPSK coherent receiver, IEEE Photon. Technol. Lett., vol. 20, pp. 1733-1735, 2008.

[4] S.H. Chang et al.: Impact of quadrature imbalance in optical coherent QPSK receiver, IEEE Photon. Technol. Lett., vol. 21, pp. 709-711, 2009.

[5] C.S. Petrou et al::Quadrature imbalance compensation for PDM QPSK coherent optical systems, IEEE Photon. Technol. Lett., vol. 21, pp. 1876-1878, 2009.

[6] B. Razavi: Design considerations for direct-conversion receivers, IEEE Trans. Circuits Syst. II, Analog Digit. Signal Process., vol. 44, pp. 45-52, 1997.

[7] J. Proakis: Digital Communications, Chap. 5 and Chap. 11, McGraw-Hill, $4^{\text {th }}$ edition, 2000. 